\title{
The influence of systemic glucocorticoid therapy upon the risk of non-serious infection in older patients with rheumatoid arthritis: a nested case-control study
}

\author{
W G Dixon, ${ }^{1,2}$ A Kezouh, ${ }^{2}$ S Bernatsky, ${ }^{3}$ S Suissa ${ }^{2}$
}

\section{- Supplementary appendix A is published online only in file 1 . To view this file please visit the journal online (http://ard.bmj. com) \\ ${ }^{1}$ Arthritis Research UK Epidemiology Unit, Manchester Academic Health Science Centre,The University of Manchester, Manchester, UK ${ }^{2}$ Centre for Clinical Epidemiology, Lady Davis Institute - Jewish General Hospital, McGill University, Montreal, Canada ${ }^{3}$ Division of Clinical Epidemiology, McGill University Health Centre, Montreal, Canada}

\section{Correspondence to} Dr W G Dixon, Arthritis Research UK Epidemiology Unit, The University of Manchester, Stopford Building, Oxford Road, Manchester M13 9PT, UK; will.dixon@manchester.ac.uk

Accepted 2 January 2011 Published Online First

1 February 2011

\section{ABSTRACT \\ Background Glucocorticoid therapy is strongly associated with an elevated risk of serious infections in patients with rheumatoid arthritis (RA). The association between glucocorticoids and common non-serious infections (NSI) is not well studied.}

Methods A cohort of 16207 patients with RA aged over 65 years was assembled using administrative data from Quebec. Glucocorticoid and disease-modifying antirheumatic drug (DMARD) therapy were identified from drug dispensing records. NSI cases were defined as first occurrence of a community physician billing code for infection or community-dispensed anti-infectives. A nested case-control analysis was performed considering drugs dispensed within 45 days of the index date, adjusting for age, sex, markers of disease severity, DMARD and comorbidity.

Results For 13634 subjects, a NSI occurred during 28695 person-years of follow-up, generating an incidence rate of $47.5 / 100$ person-years. The crude rate of NSI in glucocorticoid-exposed and unexposed person time was 52.4 and $38.8 / 100$ person-years, respectively. Glucocorticoid therapy was associated with an adjusted RR of 1.20 (95\% Cl 1.15 to 1.25). A dose response was seen, the adjusted RR increasing from $1.10 \mid<5 \mathrm{mg}$ prednisolone/day) to 1.85 for doses greater than 20 $\mathrm{mg} /$ day. All glucocorticoid risk estimates (including $<5$ $\mathrm{mg} /$ day) were higher than that seen for methotrexate (adjusted RR 1.00; 0.95 to 1.04).

Conclusion Glucocorticoid therapy is associated with an increased risk of NSI. The magnitude of risk increases with dose, and is higher than that seen with methotrexate, although residual confounding may exist. While the RR is low at 1.20 , the absolute risk is high with one additional infection seen for every 13 patients treated with glucocorticoids for 1 year.

Glucocorticoid therapy was introduced as a treatment for patients with rheumatoid arthritis (RA) nearly 60 years ago. ${ }^{1}$ Approximately one third of patients with RA are current users, and two thirds of patients have ever used steroids. ${ }^{2}$ Although glucocorticoid therapy improves the symptoms of active RA $^{3}$ and modifies disease progression, ${ }^{4}$ there have long been concerns about safety. One of the major risks associated with glucocorticoid therapy is infection, along with others including cardiovascular disease, diabetes and osteoporosis. ${ }^{5}$

The association between glucocorticoid therapy and serious infection (generally defined as infection leading to hospitalisation, intravenous antibiotics, significant loss of function or disability or death) is now well established in observational studies. ${ }^{6-15}$ Randomised clinical trials are often too small and thus underpowered to detect risks of serious infections. The risk of infection is dose-dependent, 101113 although it is not clear if there is a threshold below which glucocorticoid therapy is safe. Comparisons with the risk associated with other traditional disease-modifying antirheumatic drugs (DMARD) suggest glucocorticoid therapy has a higher RR. ${ }^{6-12}$

To date, little research has explored the association between glucocorticoid therapy and non-serious infection (NSI) in patients with RA. Although these events are not life-threatening, the burden of NSI is high. Non-serious respiratory infections account for 300-400 general practice consultations annually per 1000 registered patients in the UK. ${ }^{16}$ Even a modest increase in the RR of NSI with glucocorticoid therapy could therefore represent a large increase in the absolute or attributable risk and a significant health burden.

Our primary aim was to test the hypothesis that systemic glucocorticoid therapy is associated with an increased risk of NSI in patients with RA compared with patients with RA not treated with glucocorticoids using a nested case-control analysis. Secondary aims were to estimate the attributable risk associated with glucocorticoids, explore any dose-dependent risk and to compare the glucocorticoid-associated risk with the risk associated with other DMARD treatments.

\section{METHODS}

A cohort study and nested case-control analysis was conducted to examine the influence of systemic glucocorticoid therapy upon the risk of NSI in patients with RA. Ethical approval was obtained from the McGill University Institutional Review Board.

\section{Study base}

Patients with RA were assembled from the administrative databases of the Régie de l'assurance maladie du Québec (RAMQ) and the Ministry of Health's Maintenance et Exploitation des Données pour l'Étude de la Clientèle Hospitalière (MEDECHO). The RAMO is responsible for administering universal healthcare services for the province of Québec, Canada. It contains three databases linked by an individual's unique health insurance number: a demographic database, a medical services database and a prescription database. The demographic 
database contains information on age and sex for all registered eligible healthcare beneficiaries in Québec. The medical services database contains the date and location of appointments, specialty of the treating and referring physician and the diagnostic code of the billed service (International Classification of Diseases (ICD-9) code). The prescription database contains information on dispensed outpatient prescription medications including drug name, dosage and amount dispensed, date dispensed and prescribed number of days of treatment. The prescription programme is restricted to individuals 65 years of age and older, welfare recipients and individuals without private drug insurance through their employer. The MEDECHO database records data on acute care hospital admissions for all residents of Québec. Data exist on the primary and up to 15 secondary discharge diagnoses, date of admission, duration of hospital stay and treatments received while in hospital.

\section{Study population}

The study population from which the cohort was formed consisted of all patients with RA aged over 65 years who, between 1 January 1985 and 31 December 2003, were dispensed at least one traditional or biological DMARD in the RAMQ prescription programme. RA was identified from the physician billing code (ICD-9 code 714). Eligible DMARD included methotrexate, sulfasalazine, leflunomide, hydroxychloroquine, chloroquine, azathioprine, cyclophosphamide, cyclosporine, gold compounds, mycophenolate mofetil, d-penicillamine and the anti-tumour necrosis factor (TNF) agents infliximab, etanercept and adalimumab. Cohort entry was defined as the date of first DMARD prescription following an RA diagnosis, or 1 January 1985, whichever came later. The age restriction of over 65 years was to ensure complete prescription records from RAMO for the whole population. Subjects were all followed from the cohort entry date to the earliest outcome of interest, death or the end of study period (31 December 2003). Subjects were required to have more than 3 months of eligibility in the health insurance plan before cohort entry.

\section{Definition of cases and controls}

NSI cases were defined as the first occurrence of either a physician billing code for infection or community-dispensed antiinfectives, taking the first occurrence of either as the index case. A sensitivity analysis was performed requiring cases to have both a billing code and an anti-infective, when the billing code preceded the anti-infective prescription by 1 week or less. This definition would include a higher proportion of definite infections but would miss a large number of genuine infections in which either the single billing code did not capture the infection or anti-infectives were appropriately not prescribed. The codes for infection are listed in supplementary appendix A, available online only in supplementary file 1 . Cases were therefore subjects with a first NSI (with its index date), occurring between cohort entry and 31 December 2003.

For each NSI that occurred in the cohort, we identified a 'risk set' of patients who had not yet developed a NSI by that index date, but who remained at risk. For each case, we randomly selected five controls from the risk set, matched on entry date and time in cohort. The date of NSI was designated as the index date for each case-control set. Controls could later be included as cases, and a patient acting as a control for one case could be selected again as a control for future cases during the study period. One patient could therefore act many times as a control during their follow-up, albeit at different times.

\section{Exposure}

All drugs dispensed in the 45 days before the index date were identified from the prescription database. Systemic glucocorticoid therapy (oral, intravenous or intramuscular injection) was identified using unique drug identification numbers obtained from the Drug Product Database of Health Canada. ${ }^{17}$ Current glucocorticoid exposure was categorised as yes/no, current exposure defined as any prescription dispensed within the 45-day period before the index date. Four sensitivity analyses were conducted by varying the time window for any prescription to 15 , 30, 90 and 180 days before the index date. The glucocorticoid dosage was converted to daily prednisolone equivalent dosage (PEQ), and categorised as $0,0.1-4.9,5-9.9,10-14.9,15-19.9$ and $20 \mathrm{mg}$ PEQ and above. A dose of glucocorticoid therapy was considered for oral therapy only.

\section{Statistical analysis}

Person-years of follow-up within the whole cohort were totalled to estimate the incidence rates of a first NSI. Incidence rates were also separately calculated for person time exposed or not exposed to glucocorticoid therapy. In this cohort analysis, exposed time began on the day of glucocorticoid dispensing and continued for the duration of the glucocorticoid prescription.

Within the nested case-control analysis, conditional logistic regression was used to calculate the $\mathrm{OR}$ and $95 \% \mathrm{CI}$ for infection for systemic glucocorticoid therapy, as well as DMARD. Using risk-set sampling, the estimated OR can be interpreted as a rate ratio or RR. ${ }^{18}$ Results are presented as $\mathrm{RR}$ with $95 \% \mathrm{CI}$. Sequential analyses exploring the RR conferred by glucocorticoid therapy (oral, injectable or either) were undertaken, first adjusting for age and sex, then concurrent DMARD therapy, then all a priori confounders. The association of DMARD use and NSI was also reported, adjusted for all other drugs to provide estimates of the independent effects of each drug, then further adjusted for the a priori confounders. All analyses were performed using SAS (version 9.2).

\section{Potential confounders}

Disease severity and comorbidity were both potential confounders. No direct measures of disease severity exist within $\mathrm{RAMQ}$, and therefore several surrogate markers were included in the analysis (number of rheumatologist visits in the preceding 6 months, current non-steroidal anti-inflammatory drug (NSAID) use, previous code for extra-articular RA). Comorbidity was identified from diagnoses made before the index date, using ICD-9 codes for physician encounters (inpatient or outpatient) of diabetes mellitus, chronic respiratory conditions (chronic obstructive pulmonary disease, asthma or interstitial lung disease), cancer, chronic renal disease and osteoporosis. These comorbidities were also identified from medication typically associated with its treatment (eg, insulin for diabetes). The comorbidity items were chosen because of their potential association with both glucocorticoid exposure and with the risk of infection. Other covariates included in the model were age, sex and gastric acid-suppressive drugs, given their possible association with both glucocorticoid use and infection risk. ${ }^{19}$

\section{RESULTS}

Overall, 16207 patients with RA aged $>65$ were identified. Twothirds of the cohort were women, and the mean age was 71 years (table 1). The most commonly prescribed DMARD were 
Table 1 Baseline characteristics of RA subjects at cohort entry $(\mathrm{n}=16$ 207)

\begin{tabular}{lc}
\hline Age (years, mean (SD)) & $70.9(5.9)$ \\
Female (number (\%)) & 68.3 \\
DMARD use at cohort entry* & \\
Methotrexate & 37.9 \\
Sulfasalazine & 3.3 \\
Leflunomide & 0.2 \\
Chloroquine/hydroxychloroquine & 42.9 \\
Azathioprine & 2.2 \\
Cyclophosphamide & 2.3 \\
Anti-TNF therapy & 0.01 \\
Gold & 10.6 \\
Others (ciclosporin, mycophenolate mofetil, d-penicillamine) & 2.8 \\
\hline
\end{tabular}

*Patients could receive combination disease-modifying antirheumatic drug (DMARD) therapy and contribute to more than one drug category, allowing total to exceed $100 \%$ RA, rheumatoid arthritis; TNF, tumour necrosis factor.

the antimalarials chloroquine and hydroxychloroquine (43\%) followed by methotrexate (38\%). Less than $1 \%$ of patients were treated with anti-TNF drugs, reflecting the introduction of biological therapy late in the calendar period of the study. During the study period, there were 13634 first-episode NSI during 28695 person-years of follow-up, generating an overall incidence rate of 47.5/100 person-years. The commonest site of infection was the respiratory tract. The crude rate of NSI in glucocorticoid-exposed and unexposed person time was 52.4 and 38.8/100 person-years, respectively. The 13634 patients with first episodes of NSI comprised the cases for the nested casecontrol analysis.

Cases and controls had similar age and sex profiles, despite being matched only on entry date and time in cohort (table 2). The mean age was slightly higher in the cases and controls than it was for the whole cohort. The cases had more extra-articular RA and more general practitioner and hospital specialist visits in the year before the index date. They also had a higher prevalence of all comorbidities (diabetes, chronic respiratory disease, osteoporosis, chronic renal disease and cancer), and more commonly used gastric acid suppressants.

Approximately $35 \%$ of patients had current exposure to glucocorticoid therapy. Exposure to current glucocorticoid therapy was associated with an increased risk of NSI (age and sexadjusted RR (aRR) 1.28, 95\% CI 1.23 to 1.33; table 3). Further adjustment for concurrent DMARD therapy made little difference to the estimate, although additional adjustment for the a priori confounders reduced the estimate to 1.20 (1.15 to 1.25). Adjusting the definition of current glucocorticoid exposure to prescriptions dispensed within $15,30,90$ or 180 days before the index date resulted in aRR estimates of 1.22 (1.16 to 1.28), 1.21 (1.16 to 1.26$), 1.18$ (1.13 to 1.23 ) and 1.16 (1.11 to 1.20 ), respectively.

Using the unexposed cohort event rate of $38.8 / 100$ personyears and the aRR of 1.20 , the attributable risk from glucocorticoid therapy was 7.8/100 person-years. In other words, for every 13 patients treated with glucocorticoid therapy over a 1-year period, there would be one additional NSI compared with patients not treated with glucocorticoids.

The most commonly dispensed dose of oral glucocorticoid therapy was between 5 and $9.9 \mathrm{mg}$ PEQ per day. All dose categories of oral glucocorticoid therapy appeared to have an increased RR of infection, with a noticeably higher risk at doses greater than $20 \mathrm{mg}$ PEQ per day. There appeared to be a stepwise increased risk associated with increasing dose through the dose categories, notably at 10 and $20 \mathrm{mg}$ PEO. Only $2 \%$ of
Table 2 Baseline characteristics of cases and matched controls from a base population of 16207 RA patients, 1985-2003

\begin{tabular}{|c|c|c|}
\hline & Cases & Controls* \\
\hline Number & 13634 & 68170 \\
\hline Age (years, mean (SD)) & $71.9(6.0)$ & $71.8(5.9)$ \\
\hline Sex (\% women) & 68.9 & 68.9 \\
\hline Follow-up (years, mean (SD)) & $1.2(1.5)$ & $1.2(1.5)$ \\
\hline $\begin{array}{l}\text { Number of rheumatologist visits in } 6 \text { months } \\
\text { before index date (mean (SD)) }\end{array}$ & $1.5(2.7)$ & $1.4(2.5)$ \\
\hline NSAID prescription within last 45 days & 55.2 & 55.7 \\
\hline Extra-articular RA & 0.2 & 0.1 \\
\hline \multicolumn{3}{|l|}{ DMARD prescription within past 45 days $\dagger$} \\
\hline Methotrexate & 33.3 & 33.0 \\
\hline Sulfasalazine & 2.4 & 3.0 \\
\hline Leflunomide & 0.3 & 0.3 \\
\hline Chloroquine/hydroxychloroquine & 29.4 & 30.7 \\
\hline Azathioprine & 1.9 & 1.5 \\
\hline Cyclophosphamide & 1.7 & 0.7 \\
\hline Anti-TNF therapy & 0.1 & 0.1 \\
\hline Gold & 7.1 & 6.5 \\
\hline $\begin{array}{l}\text { Others (ciclosporin, mycophenolate mofetil, } \\
\text { d-penicillamine) }\end{array}$ & 1.8 & 1.7 \\
\hline $\begin{array}{l}\text { No of hospital admissions in year preceding index } \\
\text { date (mean (SD)) }\end{array}$ & $0.5(1.0)$ & $0.4(0.8)$ \\
\hline $\begin{array}{l}\text { No of GP visits in year preceding index date (mean } \\
\text { (SD)) }\end{array}$ & $7.8(9.0)$ & $6.1(7.7)$ \\
\hline $\begin{array}{l}\text { No of hospital specialist visits (excluding } \\
\text { rheumatologist) in year preceding index date } \\
\text { (mean (SD)) }\end{array}$ & $9.9(13.1)$ & $7.6(10.8)$ \\
\hline \multicolumn{3}{|l|}{ Comorbidity (from ICD-9 or drug codes) } \\
\hline Diabetes & 9.3 & 7.8 \\
\hline Chronic respiratory disease (COPD/asthma/ILD) & 20.3 & 12.1 \\
\hline Osteoporosis & 17.9 & 15.6 \\
\hline Chronic renal disease & 20.3 & 12.1 \\
\hline Cancer & 4.3 & 2.7 \\
\hline $\mathrm{PPI} / \mathrm{H} 2$ blocker prescription within last 45 days & 27.9 & 20.0 \\
\hline
\end{tabular}

Numbers are percentages unless otherwise stated.

*Each patient within the cohort could contribute more than once to the controls. tPatients could receive combination disease-modifying antirheumatic drug (DMARD) therapy and contribute to more than one drug category.

COPD, chronic obstructive pulmonary disease; GP, general practitioner; ICD, International Classification of Diseases; ILD, interstitial lung disease; NSAID, non-steroidal antiinflammatory drug; PPI, proton pump inhibitor; RA, rheumatoid arthritis.

patients had had a systemic glucocorticoid injection in the 45 days before the index date. The association between injectable glucocorticoid therapy and infection was inconclusive, with possible effect sizes ranging from a slight protective effect to a $22 \%$ increased risk. Too few patients $(<1 \%)$ were dispensed both oral and injectable glucocorticoid therapy to enable robust effect estimates.

Current exposure to methotrexate was associated with no increased risk of infection compared with no methotrexate (aRR $1.00,0.95$ to 1.04 ; table 4). Patients currently prescribed sulfasalazine or antimalarials had a lower rate of infection than those not prescribed those drugs. Cyclophosphamide was associated with a higher risk of infection (aRR 2.14, 1.51 to 3.03). The results for anti-TNF therapy were inconclusive given the small number of patients exposed to them. Gastric acid-suppressive drug prescription (proton pump inhibitors or $\mathrm{H} 2$ blockers) in the 45 days before the index date was associated with an increased risk of NSI (aRR 1.36, 1.30 to 1.43 ).

In order to explore the validity of the event definition, we performed a sensitivity analysis by requiring any NSI to have both a billing code and an anti-infective prescription within a week of each other. The aRR of NSI for oral glucocorticoid exposure in the preceding 45 days using the stricter outcome definition was 1.09 (0.98 to 1.22$)$. 
Table 3 Effect of current glucocorticoid therapy upon non-serious infection (billing code or anti-infective prescription), stratified by mode of administration (oral only, injection only, oral or injection) and dose

\begin{tabular}{|c|c|c|c|c|c|c|}
\hline & $\begin{array}{l}\text { Cases } \\
(n=13 \text { 634) }\end{array}$ & $\begin{array}{l}\text { Controls } \\
(n=68170)\end{array}$ & $\begin{array}{l}\text { Crude RR } \\
(95 \% \mathrm{CI})\end{array}$ & $\begin{array}{l}\text { RR, adjusted for age } \\
\text { and sex only ( } 95 \% \mathrm{CI})\end{array}$ & $\begin{array}{l}\text { RR, adjusted for age and } \\
\text { sex and DMARD (95\% CI) }\end{array}$ & Adjusted RR* $(95 \% \mathrm{CI})$ \\
\hline $\begin{array}{l}\text { Oral glucocorticoid exposure } \\
\text { within past } 45 \text { days }\end{array}$ & 37.9 & 32.5 & $1.28(1.23$ to 1.33$)$ & $1.28(1.23$ to 1.33$)$ & $1.28(1.23$ to 1.34$)$ & $1.20(1.15$ to 1.25$)$ \\
\hline \multicolumn{7}{|c|}{ Average daily dose of glucocorticoid therapy } \\
\hline$<5 \mathrm{mg}$ PEQ & 3.2 & 3.1 & $1.13(1.01$ to 1.25$)$ & $1.13(1.01$ to 1.25$)$ & $1.13(1.02$ to 1.26$)$ & $1.10(0.99$ to 1.22$)$ \\
\hline $5-9.9 \mathrm{mg}$ & 17.8 & 17.1 & $1.15(1.09$ to 1.20$)$ & $1.15(1.09$ to 1.20$)$ & $1.15(1.10$ to 1.21$)$ & $1.10(1.04$ to 1.16$)$ \\
\hline $10-14.9 \mathrm{mg}$ & 9.5 & 7.9 & $1.33(1.25$ to 1.42$)$ & $1.33(1.25$ to 1.42$)$ & $1.34(1.26$ to 1.44$)$ & $1.25(1.17$ to 1.34$)$ \\
\hline $15-19.9 \mathrm{mg}$ & 2.7 & 2.1 & $1.41(1.25$ to 1.58$)$ & $1.41(1.25$ to 1.58$)$ & $1.40(1.25$ to 1.58$)$ & $1.26(1.12$ to 1.42$)$ \\
\hline$\geq 20 \mathrm{mg}$ & 4.7 & 2.3 & $2.33(2.11$ to 2.56$)$ & 2.33 (2.11 to 2.56$)$ & $2.27(2.06$ to 2.50$)$ & 1.85 (1.68 to 2.05$)$ \\
\hline $\begin{array}{l}\text { Systemic glucocorticoid } \\
\text { injection within past } 45 \text { days }\end{array}$ & 2.2 & 1.8 & $1.18(1.04$ to 1.34$)$ & $1.18(1.04$ to 1.34$)$ & $1.18(1.04$ to 1.34$)$ & 1.07 (0.94 to 1.22$)$ \\
\hline \multicolumn{2}{|c|}{ Any glucocorticoid: oral or injection 39.2} & 33.7 & $1.28(1.23$ to 1.33$)$ & $1.28(1.23$ to 1.33$)$ & $1.29(1.24$ to 1.34$)$ & $1.19(1.14$ to 1.24$)$ \\
\hline
\end{tabular}

Numbers are percentages unless otherwise stated.

${ }^{*}$ Adjusted for all a priori confounders listed in table 2.

DMARD, disease-modifying antirheumatic drug; PEQ, prednisolone equivalent dose.

Table 4 Crude and aRR of non-serious infection, by current DMARD use

\begin{tabular}{|c|c|c|c|c|}
\hline & $\begin{array}{l}\text { Cases } \\
(n=13 \text { 634) }\end{array}$ & $\begin{array}{l}\text { Controls } \\
(n=68 \text { 170) }\end{array}$ & aRR, model 1 (95\% Cl) & aRR, model 2 (95\% Cl) \\
\hline \multicolumn{5}{|l|}{ Current DMARD use } \\
\hline Methotrexate & 33.3 & 33.0 & $0.98(0.94$ to 1.03$)$ & 1.00 (0.95 to 1.04$)$ \\
\hline Sulfasalazine & 2.4 & 3.0 & 0.81 (0.72 to 0.91$)$ & 0.79 (0.70 to 0.89$)$ \\
\hline Leflunomide & 0.3 & 0.3 & 1.07 (0.76 to 1.49 ) & 1.00 (0.71 to 1.39 ) \\
\hline $\mathrm{CO} / \mathrm{HCO}$ & 29.4 & 30.7 & $0.91(0.87$ to 0.96$)$ & 0.93 (0.89 to 0.98$)$ \\
\hline Azathioprine & 1.9 & 1.5 & $1.13(0.99$ to 1.31$)$ & 1.05 (0.91 to 1.22$)$ \\
\hline Cyclophosphamide & 1.7 & 0.7 & 2.86 (2.05 to 3.99$)$ & $2.14(1.51$ to 3.03$)$ \\
\hline Gold & 7.1 & 6.5 & $1.13(1.04$ to 1.23$)$ & $1.08(0.99$ to 1.18$)$ \\
\hline Anti-tumour necrosis factor therapy & 0.1 & 0.1 & 1.41 (0.83 to 2.39$)$ & 1.48 (0.87 to 2.52$)$ \\
\hline Others & 1.8 & 1.7 & $1.08(0.93$ to 1.25$)$ & $1.07(0.92$ to 1.25$)$ \\
\hline
\end{tabular}

Numbers are percentages unless otherwise stated.

aRR, adjusted RR, comparing current drug use with no current drug use; $\mathrm{CQ} / \mathrm{HCO}$, chloroquine/hydroxychloroquine; DMARD, diseasemodifying antirheumatic drug; model 1, adjusted for all other drugs in the table and glucocorticoids; model 2, model 1 plus further adjusted for all other a priori confounders listed in table 2.

\section{DISCUSSION}

Exposure to current oral glucocorticoid therapy was associated with a $20 \%$ increased risk of infection. The magnitude of this increased risk was slightly higher than that seen with methotrexate. This risk can be expressed as a number needed to harm, in which for every 13 patients treated with glucocorticoid therapy over a 1-year period (assuming a constant hazard), there was one additional NSI. Whereas the risk appeared greater with any current oral glucocorticoid therapy compared with none, there was a dose-response relationship, and the risk was clearly greater at daily doses above $20 \mathrm{mg}$ PEQ per day (aRR 1.85, 1.68 to 2.05).

These results replicate the findings of another Canadian administrative database study, which found an aRR of 1.15 (1.11 to 1.19) for mild (ie, non-serious) infections comparing oral glucocorticoid therapy with no glucocorticoid or DMARD therapy. ${ }^{7}$ Allowing for some differences between the studies, this near-identical result adds weight to the observed association, although similar biases in the two studies cannot be ruled out. Many studies of serious infection exist, with RR estimates for all-site serious infection ranging from 1.5 to $2.5 .^{6-10}$ Similar estimates exist for site-specifici ${ }^{11} 12$ and organism-specific 131420 serious infection. Although the magnitude of $R R$ is not as high with non-serious compared with serious infections, the baseline rate of NSI is much greater. This means that, despite the lower RR for NSI, the actual number of additional cases attributed to glucocorticoid therapy is greater for non-serious compared with serious infection.
The strengths of this study include its size. We were able to identify over 13000 patients with an infection in a patient population of over 16000 patients. Although this meant that individual subjects were selected more than once as controls, this is necessary within nested case-control analyses to avoid the introduction of bias. ${ }^{21}$ The administrative setting meant that there was no selection bias, as we were able to study the whole population over 65 years. Indeed, this older population is at higher background risk of infection and is of particular clinical interest.

Limitations do exist in this administrative database study. Patients with RA were identified using billing codes, introducing the possible misclassification of patients with other musculoskeletal diseases. The additional requirement for a DMARD prescription should, however, minimise this problem. Glucocorticoid exposure was ascertained from dispensary data. Once dispensed, it is possible that patients were non-adherent. This could include patients not taking medication that was dispensed, or alternatively taking cached medication. Such patient decisions may be influenced by disease activity. Any resultant misclassification of the exposure could be differential in either direction and could bias the result either towards or away from the null. We have considered the influence of only current glucocorticoid exposure upon the risk of NSI. Although more complex methods exist to model the relationship flexibly between time-varying dose and outcome, our analysis methodology matches that of previous studies, thereby making the results more comparable. Furthermore, it makes biological sense that 
glucocorticoid therapy more than 45 days before an infection would have much less influence upon infection risk than recent or current therapy.

The low RR of 1.20 is in the range of values that might reasonably be explained by bias. ${ }^{22}$ Clinicians may prescribe antiinfectives at different thresholds to patients under treatment with glucocorticoid or DMARD therapy. For example, a patient with a sore throat and mild temperature may be more likely to receive anti-infectives if immunosuppressed. This would increase the relative proportion of cases exposed to glucocorticoid and DMARD therapy, and might explain some of the observed effect. We cannot assess whether this explains the whole effect, although it seems unlikely. Another concern is confounding by disease severity, in which patients with high disease activity and thus at higher risk of infection ${ }^{9}$ are more likely to be prescribed glucocorticoid therapy. Disease severity is not measured directly in $\mathrm{RAMQ}$, so we used surrogate markers that were available, including the number of rheumatologist visits in the preceding 6 months and concomitant NSAID use. Because these are imperfect surrogates, residual confounding will exist. We can, nonetheless, explore whether different levels of disease severity in glucocorticoid-exposed and unexposed patients can wholly explain the observed relationship (ie, reduce the aRR from 1.2 to 1 ) using the 'rule-out approach'. ${ }^{23}$ Assuming a baseline prevalence of 'high disease severity' of $20 \%$ in the whole cohort and a RR of NSI of 1.27 for this confounder, ${ }^{9}$ disease severity cannot explain the observed aRR of 1.20. Even if we assume a doubling of NSI for the $20 \%$ of patients with 'high disease severity', this unmeasured confounder would have to be nearly four times more common in glucocorticoid-exposed patients to explain fully the observed aRR of 1.2. However, while theoretically possible, no binary measure of disease severity has yet been reported with such a strong association. Finally, there may be confounding by contraindication, in which a patient with high comorbidity is considered too 'high risk' for traditional DMARD therapy and is instead prescribed glucocorticoid therapy. We attempted to adjust for this in the analysis.

The results of the dose-dependent analysis suggest that the increased risk of NSI exists at a daily dose of 5-10 mg PEQ. It may even be present at doses less than $5 \mathrm{mg}$, although this result was inconclusive ( $\mathrm{RRR} 1.10,0.99$ to 1.22). It is interesting to compare the effect sizes between oral glucocorticoid therapy and methotrexate. At any dose, glucocorticoid therapy was associated with a higher RR of NSI compared with that seen with methotrexate. This is important to note as some clinicians see steroids as the 'safer choice' for older patients. The preferential prescription of glucocorticoid therapy over methotrexate may therefore be misguided with respect to infections, even at low doses. Any comparison between the two drugs must, of course, take account of all adverse drug reactions and not just NSI. Nonetheless, patients treated with glucocorticoid therapy have repeatedly been shown to have higher rates of serious infection than patients treated with methotrexate in observational studies. ${ }^{6-12}$ Older patients are also at high risk of other glucocorticoid-associated adverse events such as osteoporosis and cardiovascular disease. The comparative safety of glucocorticoid and DMARD therapy, in particular in older patients and patients with existing comorbidity, warrants careful consideration.

In summary, we have shown that glucocorticoid therapy is associated with a modest relative, but high attributable, risk of NSI. While some of this association may be explained by residual confounding, clinicians should nonetheless consider carefully the risks of glucocorticoid therapy in older patients, even at low doses.

Funding WGD was partly supported by a travel award from the Dickinson Trust Scholarship Fund, Central Manchester Foundation Trust. The database acquisition was funded by a grant to SS from the Canadian Institutes of Health Research (CIHR) and the Canadian Foundation for Innovation (CFI).

Contributors Study design: WGD, SS; data acquisition: SS, SB; manuscript preparation: WGD, SB, SS; statistical analysis: AK.

Ethics approval This study was conducted with the approval of the McGill University Institutional Review Board.

Provenance and peer review Not commissioned; externally peer reviewed.

\section{REFERENCES}

1. Hench PS, Kendall EC, Slocumb CH, et al. Effects of cortisone acetate and pituitary $\mathrm{ACTH}$ on rheumatoid arthritis, rheumatic fever and certain other conditions. Arch Intern Med (Chic) 1950:85:545-666.

2. Caplan L, Wolfe F, Russell AS, et al. Corticosteroid use in rheumatoid arthritis: prevalence, predictors, correlates, and outcomes. J Rheumatol 2007;34:696-705.

3. Gøtzsche PC, Johansen HK. Meta-analysis of short-term low dose prednisolone versus placebo and non-steroidal anti-inflammatory drugs in rheumatoid arthritis. BMJ 1998:316:811-18.

4. Kirwan JR, Bijlsma JW, Boers M, et al. Effects of glucocorticoids on radiological progression in rheumatoid arthritis. Cochrane Database Syst Rev 2007;1:CD006356.

5. Hoes JN, Jacobs JW, Verstappen SM, et al. Adverse events of low- to mediumdose oral glucocorticoids in inflammatory diseases: a meta-analysis. Ann Rheum Dis 2009;68:1833-8.

6. Bernatsky S, Hudson M, Suissa S. Anti-rheumatic drug use and risk of serious infections in rheumatoid arthritis. Rheumatology (Oxford) 2007;46:1157-60.

7. Lacaille D, Guh DP, Abrahamowicz M, et al. Use of nonbiologic disease-modifying antirheumatic drugs and risk of infection in patients with rheumatoid arthritis. Arthritis Rheum 2008;59:1074-81.

8. Franklin J, Lunt M, Bunn D, et al. Risk and predictors of infection leading to hospitalisation in a large primary-care-derived cohort of patients with inflammatory polyarthritis. Ann Rheum Dis 2007;66:308-12.

9. Doran MF, Crowson CS, Pond GR, et al. Predictors of infection in rheumatoid arthritis. Arthritis Rheum 2002;46:2294-300.

10. Smitten AL, Choi HK, Hochberg MC, et al. The risk of hospitalized infection in patients with rheumatoid arthritis. J Rheumatol 2008;35:387-93.

11. Wolfe F, Caplan L, Michaud K. Treatment for rheumatoid arthritis and the risk of hospitalization for pneumonia: associations with prednisone, disease-modifying antirheumatic drugs, and anti-tumor necrosis factor therapy. Arthritis Rheum 2006;54:628-34

12. Edwards CJ, Cooper C, Fisher D, et al. The importance of the disease process and disease-modifying antirheumatic drug treatment in the development of septic arthritis in patients with rheumatoid arthritis. Arthritis Rheum 2007:57:1151-7.

13. Strangfeld A, Listing J, Herzer P, et al. Risk of herpes zoster in patients with rheumatoid arthritis treated with anti-TNF-alpha agents. JAMA 2009:301:737-44.

14. Brassard P, Kezouh A, Suissa S. Antirheumatic drugs and the risk of tuberculosis. Clin Infect Dis 2006:43:717-22.

15. Wolfe F, Michaud K, Anderson J, et al. Tuberculosis infection in patients with rheumatoid arthritis and the effect of infliximab therapy. Arthritis Rheum 2004:50:372-9.

16. Ashworth $\mathbf{M}$, Latinovic R, Charlton J, et al. Why has antibiotic prescribing for respiratory illness declined in primary care? A longitudinal study using the General Practice Research Database. J Public Health (Oxford) 2004;26:268-74.

17. Health Canada. Drug Product Database Online Query, 2010. http://webprod.hc-sc. gc.ca/dpd-bdpp/index-eng.jsp (accessed 23 Apr 2010).

18. Rodrigues L, Kirkwood BR. Case-control designs in the study of common diseases: updates on the demise of the rare disease assumption and the choice of sampling scheme for controls. Int J Epidemiol 1990;19:205-13.

19. Laheij RJ, Sturkenboom MC, Hassing RJ, et al. Risk of community-acquired pneumonia and use of gastric acid-suppressive drugs. JAMA 2004;292:1955-60.

20. Wolfe F, Michaud K, Chakravarty EF. Rates and predictors of herpes zoster in patients with rheumatoid arthritis and non-inflammatory musculoskeletal disorders. Rheumatology (Oxford) 2006:45:1370-5.

21. Lubin JH, Gail MH. Biased selection of controls for case-control analyses of cohort studies. Biometrics 1984; 40:63-75.

22. Shapiro $\mathbf{S}$. Bias in the evaluation of low-magnitude associations: an empirical perspective. Am J Epidemiol 2000;151:939-45.

23. Schneeweiss S. Sensitivity analysis and external adjustment for unmeasured confounders in epidemiologic database studies of therapeutics. Pharmacoepidemiol Drug Saf 2006;15:291-303. 